for fast runs. The matrix indicates that medium runs are followed by medium runs and slow runs are most often followed by slow runs. Again, one-fifth of the slow runs were followed by extinction. DISCUSSION

In the analysis, two facts stand out: running fast forestalls extinction and running slowly foretells extinction. Running fast functions as a perseverative factor in that there is a high probability that the run on the next trial will also be fast. We expect that any variable which produces fast running will increase resistance to extinction. For example, short avoidance in tervals produce the fastest running speeds and also seem to increase resistance to extinction (Beecroft \& Brown, 1967). Running slowly functions as a cessative factor in that the probability that extinction will occur on the very next trial is fairly sizable. We expect that any variable which produces slow running will decrease resistance to extinction. We have shown that a retention interval decreases resistance to extinction (cf. Beecroft \& Fisher, 1970 , for details not touched upon in this paper), but we are pretty much in the dark about other variables correlated with slow running. However, the mechanism whereby slow running is related to extinction can be conjectured to be that in running slowly the animal learns the avoidance contingency is no longer in effect.

The conventional interpretation of avoidance extinction is that avoidance extinction occurs when conditioned fear extinguishes to the point that it no longer suffices to motivate the instrumental behavior. Without an independent measure of fear, the conventional theory has little or no predictive value. However, if running speed is taken to be an index of fear, then the conventional interpretation reduces to an assertion that an animal whose fear has more or less extinguished, as evidenced by slow running, will soon cease to run, the very point the transition probability matrix establishes without introducing the fear concept at all.

\section{REFERENCES}

BEECROFT, R. S. \& BOUSKA, S. A Acquisition of avoidance running in the rat. Psychonomic Science, 1967, 9, 163-164.

BEECROFT, R. S., BOUSKA, S. A., \& FISHER, B. G. Punishment intensity and self-punitive behavior. Psychonomic Science, 1967, 8, 351-352.

BEECROFT. R. 'S. \& BROWN, J. $S$ Punishment following escape and avoidance training. Psychonomic Science, 1967. 8, 349-350.

BEECROFT, R, S., \& FISHER, B. G. Avoidable punishment and self-punitive behavior. Psychonomic Science, 1969, 15, 243-244.

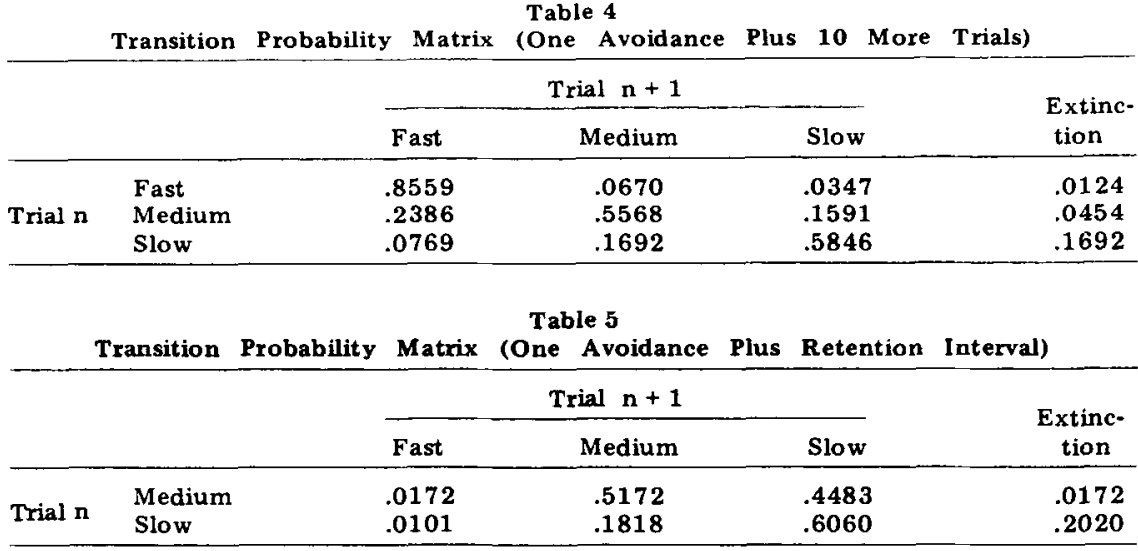

BEECROFT, R. S., \& FISHER, B. G. Retention of avoidance running. Psychonomic Science, 1970, 20, 28-29.

BEECROFT, $R$. S FISHER, B G \& BOUSKA, S. A. Punishment continuity and self-punitive behavior. Psychonomic
Science, 1967, 9, 127-128

BROWN, J. S., MARTIN, R. C., \& MORROW, M. W. Self-punitive behavior in the rat: Facilitative effects of punishment on resistance to extinction. Journal of Comparative \& Physiological

Psychology, 1964, 57, 127-133.

\title{
Postdiscrimination gradients as a function of variation in reward schedules*
}

\author{
MELVIN H. MARX and JIM McLEAN \\ University of Missouri, Columbia, Mo. 65201
}

Six pigeons were discrimination trained on wavelength stimuli and tested for postdiscrimination generalization gradients. Three were trained on Mult FI 30-sec (.50 reinforcements) extinction and three on VI 60-sec as a control. No peak shifts were found in the FI-trained birds. The VI-trained birds failed to show consistent peak shifts, a fact which suggests that the number of $\mathrm{S}+$ days provided prior to discrimination training may be an effective variable in the production of peak shift.

There are very few studies in the literature which have investigated the shape of the postdiscrimination gradient (PDG) following variation of the schedule of reward associated with $\mathrm{S}+$. Most studies provide VI 1-min schedule of reward for responding to $\mathrm{S}+$, and the most common effect observed in the PDG is peak shift. The purpose of this study was to determine if peak shift occurs in the PDG following Mult FI 30-sec (.50) extinction discrimination training. The

*This research was supported in part by Grants GB-5853 and GB-8506 from the National Science Foundation and by Research Career Award 1-K-6-MH 22023 to the first author from the National Institute of Mental Health. designation, FI $30-\sec (.50)$, indicates that the schedule of reward was basically an FI $30-$ sec schedule, but only half of the presentations of $S+$ were rewarded. The purpose of rewarding only half of the $S+$ periods was to provide a reinforcement density comparable to that provided by the VI 60 -sec schedule and, in addition, to guarantee enough resistance to extinction to make a gradient determination possible.

Besides the fixed-interval discrimination group, a control group of Ss was trained with the usual VI 60 -sec schedule associated with $\mathrm{S}+$. The discrimination was trained between $550 \mathrm{~nm} \mathrm{(S+)} \mathrm{and} 570 \mathrm{~nm}$ (S-). 


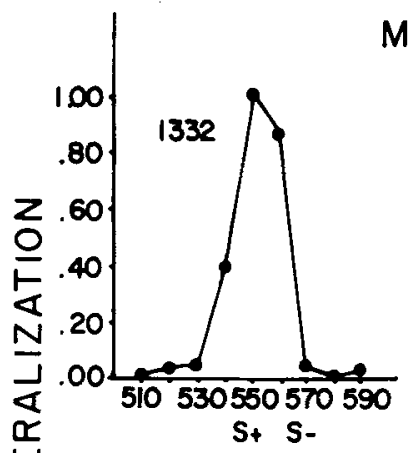

MULT. FI-30 (.50) EXT.
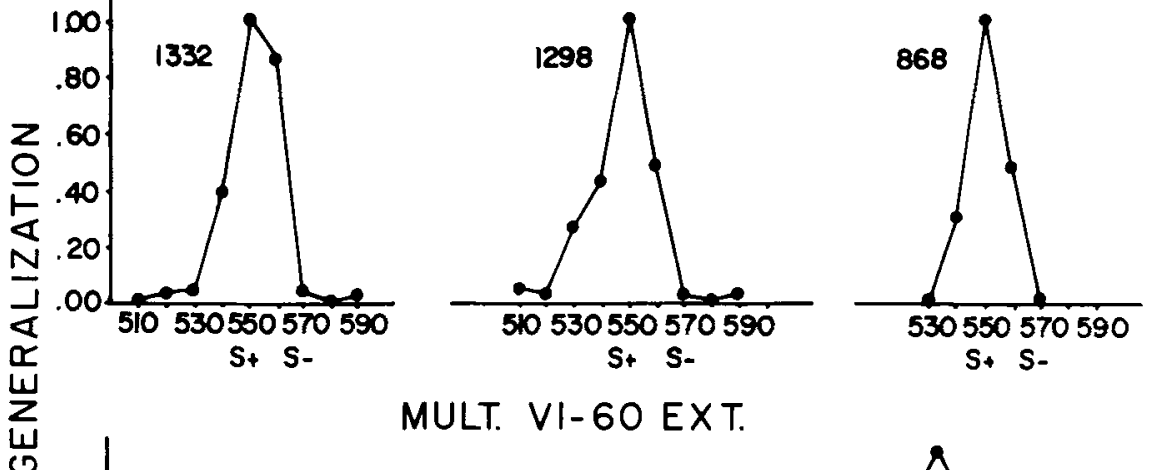

MULT. VI-60 EXT.
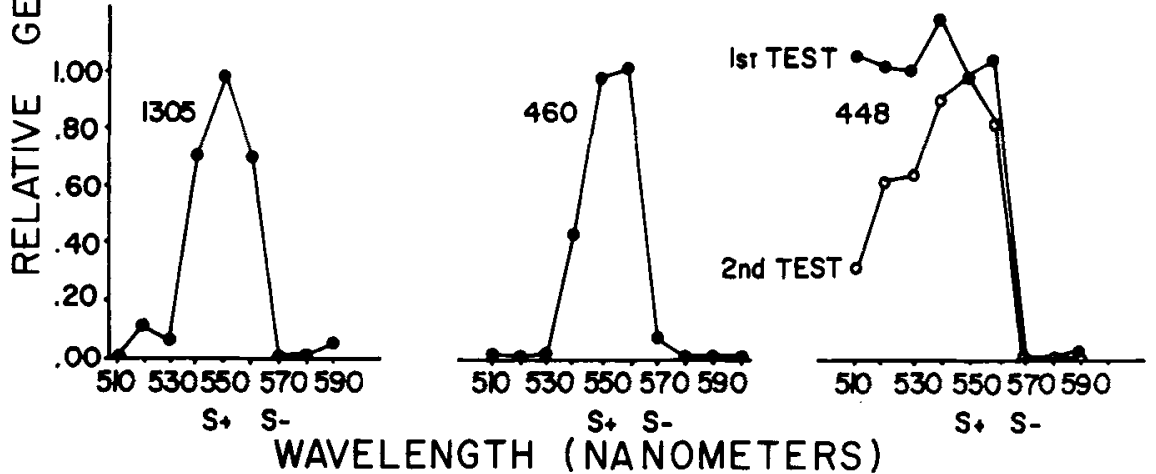

Fig. 1. Relative generalization gradients for FI- and VI-trained birds.

SUBJECTS

The Ss were six male white Carneau pigeons, 6 months to 1 year of age. They were divided into two groups of three pigeons each and were maintained at $80 \%$ of their ad lib body weights.

\section{APPARATUS}

The experimental chamber was $12 \times 12 \times 15$ in. and was located in a ventilated refrigerator shell. A 2-in.-square grain magazine on one wall afforded reinforcements. A white overhead light and colored keylights were used. The 1-in.-diam key was placed 3 in. above the food magazine itself, $3 \frac{3}{4} \mathrm{in}$. above the floor. The key was illuminated by nine wavelengths (510-590 nm). A Bausch and Lomb microscope illuminator provided the keylight, which, in addition to passing through one of the monochromatic filters, also passed through a collimating lens and a Corning K-2 auxiliary filter to remove side bands in the ultraviolet region.

Stimulus presentations, timeouts, and reinforcements were automatically programmed with orthodox operant switching devices.

\section{PROCEDURE}

One to 3 days were required to magazine and key shape the various pigeons. On the first day upon which the keypeck occurred, 30 CRF reinforcements were provided. On the second day 30 more reinforcements on the CRF schedule were given. A 10-sec blackout then separated each reinforcement. During these 2 days, the key was illuminated with $550 \mathrm{~nm}$. On the third day of training, FI or VI training was provided for responding to $\mathrm{S}+$. Since training differed from the third day on, each group will be described separately.

\section{FI Discrimination Training}

During the first session of FI training to $\mathrm{S}+$, the FI was gradually changed from FI 10-sec to FI 30-sec over the course of the first 20 trials. Following this session, there were five additional sessions of FI 30-sec training to $\mathrm{S}+$. Each of these sessions consisted of 30 trials, and a 10-sec blackout was provided at the termination of each trial. There followed 5 days of FI 30 -sec $(.50)$ training to S+. Each of these sessions consisted of 60 trials. Discrimination was then begun between $550 \mathrm{~nm}(\mathrm{~S}+)$ and $570 \mathrm{~nm}(\mathrm{~S}-)$. Each of the discrimination sessions consisted of 30 presentations of $\mathrm{S}+$ and 30 presentations of S-.

\section{Discrimination Training}

The VI training condition consisted of 6 days of VI 30 -sec to $S+, 6$ days of VI 60 -sec training to $\mathrm{S}+$, and 6 days of discrimination training. There were 30 trials per day during the VI $30-\mathrm{sec}$ period, 60 trials during the VI $60-\mathrm{sec}$ period, and 60 trials during the discrimination session ( $30 \mathrm{~S}+$ and 30 S-).
Generalization Test

Discrimination training continued for 5 days, and a generalization test was conducted on the sixth day. The generalization test, preceded by a 20-trial warm-up, consisted of presentation of the nine generalization test stimuli $(510,520,530,540,550$, $560,570,580$, and $590 \mathrm{~nm}$ ). Each stimulus was presented nine times, and the sequence of presentations was blocked so that all stimuli occurred before any stimulus occurred for a second time. Each trial of the generalization test was $30 \mathrm{sec}$ in length, and a 10-sec blackout was provided between trials.

RESULTS AND DISCUSSION

The generalization gradients for the FI and VI groups are shown in the six graphs. None of the FI-trained animals displayed peak shift in the gradient of stimulus generalization. Thus, the answer to the primary experimental question is, no, FI $30 \mathrm{sec}$ does not produce peak shift in the PDG, at least not under our experimental conditions (.50 reinforcement, etc.).

Two of the VI Ss showed shifts in the modal value of responding: one toward S+, with the peak occurring at $560 \mathrm{~nm}$, while the other displayed the strangest gradient ever obtained in our laboratory. S 448's rule of responding (over two tests) appears to have been to respond until $S-$ is presented on the key.

The failure of the VI Ss consistently to show a peak at $540 \mathrm{~nm}$ suggests that peak shift is a function of the number of days of $S+$ training provided prior to beginning the discrimination training. With small numbers of days - say 0 to 6 or 7-peak shift is obtained; but with a larger number of sessions, peak shift is not obtained. In other words, learning a discrimination between two stimuli consists of two stages, or two components. The bird must learn (1) what stimulus to respond to and (2) how to respond to that stimulus. Furthermore, peak shift is obtained only if we force the pigeon to learn both of these components at the same time. Thus, if we first teach the bird the schedule of reward, and then begin the discrimination after this is well learned, we should not expect to observe peak shift. Similarly, if we taught the bird what stimuli to respond to-by using a CRF schedule of reward to $\mathrm{S}+$, for example-and then made the schedule change to VI $60 \mathrm{sec}$, we should not expect peak shift either. As a matter of fact, peak shift is not obtained under such conditions (McLean, 1970). REFERENCE

McLEAN, J. F. Fark shift: Effects of reward schedule and $\mathrm{S}-$ duration. Unpublished $M$ aster's thesis, stanford University, 1970. 